\title{
Fracture behavior of single crystal silicon with thermal oxide layer
}

\author{
$\operatorname{AUTHOR}(S):$ \\ Tsuchiya, Toshiyuki; Miyamoto, Kenji; Sugano, Koji; \\ Tabata, Osamu
}

\section{CITATION:}

Tsuchiya, Toshiyuki ... [et al]. Fracture behavior of single crystal silicon with thermal oxide layer. Engineering Fracture Mechanics 2016, 163: 523-532

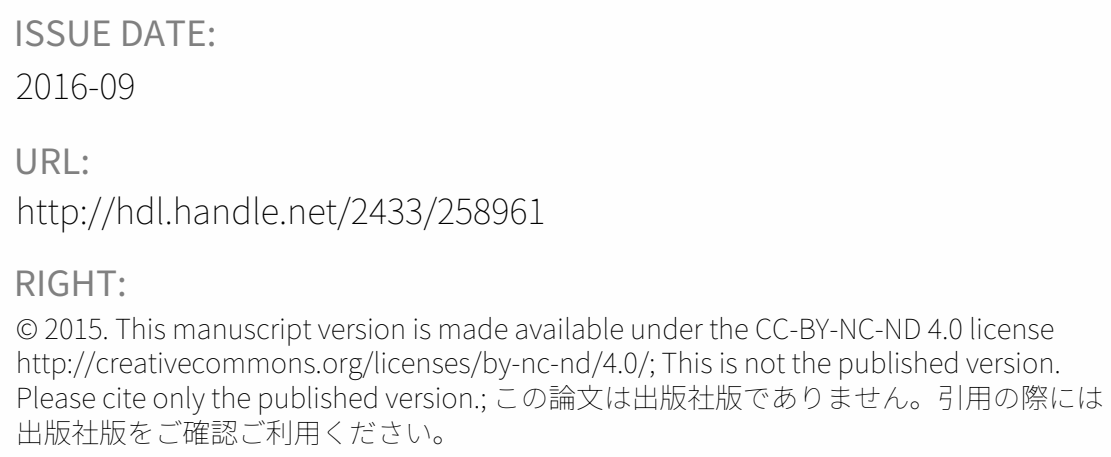




\section{Fracture behavior of single crystal silicon with thermal oxide layer}

Toshiyuki Tsuchiya, Kenji Miyamoto, Koji Sugano, Osamu Tabata

Department of Micro Engineering, Kyoto University

Kyoto-University-Katsura C3, Nishikyo-ku, Kyoto 615-8540, JAPAN

Corresponding Author: Toshiyuki Tsuchiya (tutti@me.kyoto-u.ac.jp)

\section{Abstract}

This paper reports on the effect of oxidation on fracture behavior of single crystal silicon (SCS). SCS specimens were fabricated from (100) silicon-on-insulator wafer with 5- $\mu \mathrm{m}$-thick device layer and oxide layer were thermally grown. Quasi-static tensile testing of as-fabricated, oxidized and oxidized layer removed specimens was performed. The fracture origin location transited from the surface to silicon/oxide interface and inside of silicon. The transition may be caused by surface smoothing, thickening oxide layer and formation of oxide precipitation defects in silicon during oxidation. The radius of the oxide precipitation defects was estimated, which is well agreed with the fracture-initiating crack sizes. 


\section{Introduction}

Fracture of both single crystal and polycrystalline silicon has been widely investigated for applications in microelectromechanical systems (MEMS). Failure of silicon is dominated by a fracture initiating flaw which mostly exists on the surface [1,2], the morphology and roughness of which are created during microfabrication processes, such as photolithography, wet/dry etching, thermal treatment, and wafer bonding. The relationship between the roughness, often regarded as a fracture initiating flow, and strength has been discussed extensively [3]. Control of the surfaces roughness of silicon is important for developing highly reliable micro mechanical structures.

There are various methods to improve the surface roughness and strength of silicon microstructures, such as anisotropic wet etching of silicon [4], hydrogen annealing [5], oxidation [6], and laser ablation [7]. Among them, smoothing using oxidation is simple and easy to implement to standard fabrication process. Ericson et al. reported improvement of the bending strength by oxidation and subsequent removal of oxide [6]. The improvement was caused by healing of removal of defect on the surface. Since the cantilever beams of heavily doped silicon layer was fabricated by means of dopant-selective etching and the damages were introduced by polishing process, it is required to investigate combined effect of oxidation process to the scalloping introduced by Bosch process, which is a standard process of silicon MEMS fabrication.

We have conducted tensile testing on single crystal silicon specimen oxidized after specimen fabrication to investigate the effect of an oxide surface layer on fracture of 3 - $\mu$ m-thick silicon structures using the Bosch process[8]. We reported the fracture origin transition from surface to inside of silicon possibly due to oxide precipitation. However, the etched surface was too rough to investigate the details of fracture process. In this paper, the specimen is fabricated using thicker 5 - $\mu$ m-thick silicon film with the Bosch process of finer scallops to reveal fracture behavior of oxidized silicon structures. 


\section{Experimental}

Uniaxial tensile specimen of single crystal silicon was fabricated from silicon-on-insulator (SOI) wafer of 5- $\mu \mathrm{m}$-thick device layer, using a standard SOI-MEMS fabrication process with underlayer substrate removal. The specimen is shown in fig 1 . The gauge part is $4 \mu \mathrm{m}$ wide, $5 \mu \mathrm{m}$ thick and 120 or $600 \mu \mathrm{m}$ long. The specimen surface is (100) plane and the tensile axis is $<110>$ direction, which is the standard orientation in MEMS device structures. One end of the part is fixed to the substrate and the other is connected to the large paddle for gripping. Oxidation was done after specimen release, that is, the specimen (called as as-fabricated) shown in fig. 1 was put into a RTA furnace (ULVAC, MILA-5000) and oxidized at $1100{ }^{\circ} \mathrm{C}$. The oxygen flow rate was $0.7 \mathrm{l} / \mathrm{min}$. The oxidation time was 5.5, 22.5 and 90 minutes, which correspond to the expected oxide thicknesses of 50, 100 and $200 \mathrm{~nm}$, respectively. Since it is difficult to measure the oxide thickness on the micro-scale specimen, we used these expected thicknesses. These samples denoted as 50-nm-oxide, 100-nm-oxide and 200-nm-oxide. The 200-nm-thick samples were also tested after oxide removal using hydrofluoric acid etching (called as oxide-removed). The as-fabricated, oxidized and oxide-removed specimens were tested using a custom made tensile testing system under an optical microscope[9], shown in fig. 2. The specimen-carrying substrate was aligned to the sample stage driven by piezoelectric stage (PI-polytec, P-780.20). The stage displacement was measured using a built-in LVDT sensor. The free paddle of the specimen is clamped using electrostatic gripping [2] and connected to a loadcell (Kyowa, LTS-50GA). The sample stage was covered with a small chamber connected to a temperature and humidity controlled chamber (Espec, SH-221) to control the test environment. The humidity and temperature were monitored by a sensor placed at the vicinity of the specimen. Quasi-static tensile testing was conducted at $26^{\circ} \mathrm{C}$ and $50 \% \mathrm{RH}$. The loading rate was controlled at the stage speed of $0.5 \mu \mathrm{m} / \mathrm{sec}$. The nominal tensile stress was calculated by dividing the tensile force 
by the nominal cross sectional area. The fractured surfaces were observed using field emission scanning electron microscope (Hitachi, S-4500).

The axial stress of the oxidized specimens was analyzed using the finite element method to evaluate the stress applied on both the silicon and oxide part. The gauge part of $10-\mu \mathrm{m}$-long was modelled with consideration of its symmetry. The geometry is a simple cuboid and does not take into account scalloping. The cross-sectional dimensions of the as-fabricated sample are $4 \mu \mathrm{m}$ wide and $5 \mu \mathrm{m}$ thick and the consumption of silicon by oxidation is considered for those of oxidized ones as listed in Table I. The elastic constants of silicon are anisotropic and that of silicon dioxide is isotropic, the theoretical and commonly used values are used, respectively as listed in Table II. The compressive internal stress of $460 \mathrm{MPa}$ was introduced to the oxide layer [10]. The parabolic hex elements are used and the number of nodes and elements are listed in Table III. Since automatic biased meshing was used for generating mesh, the size of mesh was not uniform but the edge lengths along the cross sections near the corner are not larger than $5 \mathrm{~nm}$. The mean fracture strength was applied at the ends and the axial stress at the symmetry plane (cross section at the middle of gauge part) was evaluated.

\section{Results}

\subsection{Stress-displacement curves}

The tensile stress-stage displacement curves of as-fabricated and 200-nm-oxide samples are shown in fig. 3. The linear plots and sudden drops of force in both the as-fabricated and oxidized specimens were observed, which indicates elastic deformation and brittle fracture of silicon and oxide films. The nominal Young's modulus $E$ was calculated by differentiating the slopes of the different length specimens, as;

$E=\left(L_{2}-L_{1}\right) /\left(1 / S_{2}-1 / S_{1}\right)$, 
where $L_{i}$ and $S_{i}(i=1,2)$ are the gauge part length and the slope of the stress-stage displacement curve, respectively, of the different length of specimens. The averaged nominal Young's moduli are shown in Table I. The theoretically calculated nominal modulus $E_{n}$ is also given by using the following equation;

$E_{n}=\frac{E_{S i} A_{S i}+E_{O X} A_{O x}}{A_{S i}+A_{O X}}$,

where $E_{x}$ and $A_{x}$ are the Young's modulus and cross sectional area of each materials and the subscript $S i$ and $O x$ indicates silicon and silicon dioxide part, respectively. The measured moduli are fluctuated but close to the theoretical values. The thicker oxide specimens show the lower nominal Young's modules. The decreasing trend agrees well with the theoretical values in Table I.

\subsection{Strength of as-fabricated specimens}

The as-fabricated specimen has the average tensile strength of $4.09 \mathrm{GPa}$. Typical fracture surfaces are shown in fig. 4. More than half of the samples fractured from the pit on the top corner as similar as our previous report [11], which may formed by a jaggy of the photoresist pattern for an etching mask. But other fracture origins were observed; either top, side, or bottom surfaces. Though these surfaces have different source of fracture; the top is surface treatment, the side is a scallop in Bosch process, and the bottom is a defect in a raw SOI wafer, the size of the defects in the sources are comparable to that on the corner. The fracture surfaces are mostly (111) plane, which is tilted by $35.4^{\circ}$ against a (110) plane perpendicular to the tensile loading axis.

\subsection{Strength of oxidized specimens}

The nominal strength of the oxidized specimens was calculated by dividing the fracture force by the total cross sectional area of each specimen in Table I. The measured nominal strength of both as-fabricated and oxidized specimens is plotted in fig. 5. The nominal strength decreased first with the growth of oxide layer to $50 \mathrm{~nm}$, but the 100-nm-oxide specimen has a higher strength than the 50-nm-oxide, a slightly lower than the as-fabricated. Then, the strength decreased again for the 
200-nm-thick-oxide specimen and it is $20 \%$ lower than the as-fabricated. The measured fracture strength of all the specimens including the oxide-removed are plotted in Weibull plots, shown in Fig. 6. The strengths of the as-fabricated and oxidized specimens are fitted to the single-parameter Weibull distribution function and fitted lines are plotted in the figure. The Weibull parameters are listed in Table IV. Note that the oxide-removed specimen does not show a smooth distribution, which is unsuitable for the single-parameter model. The Weibull modulus ranged from 5.26 to 6.42 , which is in the similar range of the tensile strength of $(110)<110>$ specimens fabricated with same process and similar conditions [11].

The fracture surfaces of the 50-nm, 100-nm, and 200-nm oxide specimens are shown in Figs. 7, 8 and 9, respectively. Almost all the specimens have a fracture surface of (111) plane. The fracture origin locations are summarized in Fig. 10. The 200-nm oxide specimen fractured mostly from the corners, whereas the other two specimen types with thinner oxide layer show the relatively large number of side surface fractures. Though it is difficult to identify the fracture origin from the FE-SEM observation, we focus on the center of the radial pattern on the fracture surface, the center of the no- and 50-nm-oxide specimen located the centers on the surface, while in the thicker oxide specimen, they seem to locate at the interface or silicon body. As we can see in Fig.9, the radial patterns can be seen in even in the oxide layer originated from inside. Therefore we estimated that most of the thicker oxide specimens $(100 \mathrm{~nm}$ and $200 \mathrm{~nm})$ fractured at the silicon body or oxide/silicon interface near the corner. Some of the 50-nm-oxide specimens that fractured from the side surface in Fig. 7 have a fracture origin at the oxide-silicon interface or oxide surface.

The oxide-removed specimens showed low average strength and large deviation. The Weibull plot shown in fig 6 indicates that there are two fracture sources; one is similar to 200-nm-oxide specimen and the other has a low strength. Some of the fracture surfaces have an apparent pit at the fracture origin, as shown in fig. 11, and it would be showing the oxide precipitation defect that is discussed 
later.

\subsection{Axial-stress in oxidized specimen}

The axial stress distribution of the gauge part at the average strength was simulated using FEM. The distribution of each specimen was extracted along the diagonal direction $\left(45^{\circ}\right)$ from the corner and is plotted against the horizontal distance from the corner in Fig. 12. The axial stress in the oxide layer is smaller than 1.3 GPa in all oxide thickness. The stress is high but it is lower than the tensile strength of $\mathrm{SiO}_{2}$ film (1.85 GPa) [12]. The oxide prepared from the single crystal silicon beam, fully oxidized using thermal oxidation the tensile testing was conducted in the air. The measured Young's modulus and fracture strain was 74 GPa and $2.5 \%$ respectively. Since the surface was smoothen by the oxidation process so the oxidized silicon sample without any post processing (dry or wet etching) has high strength.

The stress at the oxide boundary may have stress concentration but in the actual sample the corner of the boundary was rounded and the stress concentration does not occur. Since the stress of silicon in oxidized specimens is close to the strength of as-fabricated ones, the stress in silicon layer would dominate the fracture behavior of even in the oxidized specimen.

\section{Discussion}

\subsection{Fracture of as-fabricated silicon}

The as-fabricated specimens fractured not only the side or corner but also top and bottom surfaces, whereas the specimens in Ref. 8 fractured on the corner which strongly associated with the rough scallops (300 nm pitch and $100 \mathrm{~nm}$ deep) generated by the dry etching process. The specimens in this work were fabricated using a different etching machine and the scallops are fine as $120 \mathrm{~nm}$ pitch and $30 \mathrm{~nm}$ deep. Other defects on the surfaces appeared as a fracture initiating flaw.

The Weibull modulus is lower than our recent report [11] but by looking at the fitting curve, there is 
scattered point at the highest and lowest strength, which causes lower modulus, the modulus will become higher by increasing the number of specimens.

\subsection{Fracture of oxidized silicon}

Similar fracture behaviors with the previous report for the 3- $\mu$ m-thick specimens were observed [8]. The as-fabricated silicon specimens fractured from mainly the pit on the top corner and the fracture origin moved into the silicon body after thick oxidation. As we concluded in Ref. 13, the oxygen precipitation would become the fracture origin. The stress corrosion is silicon dioxide layer may also lower the strength. Since the reported tensile strength of thermally oxidized silicon in the air [12] showed high strength even in the air, we will focus on the surface roughness and the oxide precipitation in this discussion.

The radius $r(t)$ of the oxygen precipitation defects as a function of the oxidation time $t$ is described by the following equation [13],

$$
r(t)=\sqrt{2 D\left(\frac{C_{0}-C^{\prime}}{C_{p}-C^{\prime}}\right) t},
$$

where $D$ is the diffusion constant of oxygen in silicon and $C_{0}$ is the interstitial oxygen density in silicon, $C_{p}$ is the oxygen density of silicon dioxide, $C^{\prime}$ is the oxygen concentration in the silicon matrix immediately adjacent to the precipitates. The oxidation temperature was $1100{ }^{\circ} \mathrm{C}$. The $D, C^{\prime}$, $C_{p}$ and $C_{0}$ are determined to be $8 \times 10^{-11} \mathrm{~cm}^{2} / \mathrm{s}, 3 \times 10^{17}$ atoms $/ \mathrm{cm}^{3}, 4.6 \times 10^{22}$ atoms $/ \mathrm{cm}^{3}$, and 7 $\times 10^{17}$ atoms $/ \mathrm{cm}^{3}$, respectively[13,14]. The radii of the precipitates grown in the specimens are summerized in Table V. In the table, the size of the fracture initiaing defect is listed. The size was estimated using a simple fracture mechanics where an ellipsoidal shaped crack of length $2 a$ in an inifite plate fractured by tensile stress at the . The fracture stress $\sigma_{f}$ is given by,

$$
K_{I C}=\sigma_{f} \sqrt{\pi a},
$$

where $K_{I C}$ is the fracture toughness of silicon assmuming to $1 \mathrm{MPa} \sqrt{\mathrm{m}}$. The axial stress calculated from FEM analysis listed in Table IV is used as the fracture stress. The size of fracture initiating 
defects in the oxidized specimens with thicker oxide is very close to that of the precipitation size. Though it is difficult to find any apparent precipitates in the fracture surface of oxidized samples in Figs 7, 8 and 9, we observed thickening of the oxide layer near the fracture origin as seen in Fig. 9. In addtion, we observed etch pits on the surface in the oxide-removed specimens, as shown in fig. 11, which supports the existence of oxide precipitation.

The 50-nm oxide specimens, however, showed lower strength than both as-fabricated and 100-nm oxide ones. The observed specimen that fractured origin of 50-nm-oxide specimen in Fig. 7 seemed to fracture from the silicon/oxide interface or the oxide surface. The phenomenon was not observed in the previous report [8]. In addition, the existence of the fracture origin transition can be found from the Weibull analysis and fracture origin location. The 50-nm and 100-nm oxide specimens show smaller Weibull moduli and deviations from the fitting curve, which indicates the existence of the multiple types of fracture origins.

The multiple origin types can be explained by the difference in the location of the fracture origins mainly existed on the side surface and the corners. After oxidation, the strength of the specimens that fractured from side surface decreased gradually, but that fractured at the corner changed drastically by thickening the oxide layer. The drastic change of the corner fracture would be caused by the transition of the fracture origins. The as-fabricated specimen fractured from the pit caused by the jaggy of the photoresist pattern and the 200-nm-oxide specimens fractured from the oxide precipitation. The significant decrease in the strength of the 50-nm-oxide specimens may be caused by the oxidation of the corner pits. The reason of the disappearance of the fracture type at the thicker oxide specimen would be the rounding of the corner caused by oxidation. Then, the fracture from the corner of the 100-nm-oxide and 200-nm-oxide would be from the oxide precipitation, as we mentioned before.

The fracture on the side surface is related to the scallops generated by the Bosch process. Thermal 
oxidation process may generate crack like morphologies with thin ( $\sim 50 \mathrm{~nm})$ oxide layer, which acts as a fracture origin, as there is a thickened oxide layer near the fracture origin in Fig. 7.

These phenomena, especially in the 50-nm-thick specimens, would relate to the fracture and fatigue process of single crystal silicon in the air, where the native oxide may exist and stress-induced-oxide may occur. However, since there will be no effect of precipitation at low temperature at which most of the fatigue testing was conducted, low temperature oxidation process will be needed to further investigation to investigate fatigue fracture of silicon in a humid environment.

\section{Conclusion}

Quasi static tensile testing was carried out for specimens with surface oxide layer of ranged from 50 $\mathrm{nm}$ to $200 \mathrm{~nm}$. The average nominal fracture strengths of as-fabricated, 50-, 100- and 200-nm-oxide specimens were 4.09, 3.50, 3.77 and $3.27 \mathrm{GPa}$, respectively. The changes of average fracture strain were attributed to the change of fracture origin due to the surface oxidation in silicon. In the thicker oxide films, the oxide precipitation defect was thought to be a fracture initiating defect, since the estimated oxide precipitation size is comparable to the calculated size of fracture initiating defect. The knowledge would contribute for the investigation on the humidity effect on fracture and fatigue properties, in which surface oxide layer would play a significant role on their failure process.

\section{Acknowledgement}

The authors are grateful to S. Kinoshita for assistance with FE-SEM observations. Part of this work was supported by the Ministry of Education, Science, Sports and Culture, Grant-in-Aid for Young Researchers, 19676002.

\section{References}

${ }^{1}$ Izumi S, Kubodera Y, Sakai S, Miyajima H, Murakami K, Isokawa T. Influence of ICP etching 
damage on the brittle-fracture strength of single-crystal silicon. Zairyo/Journal of the Society of Materials Science 2007; 56 (10): 920-925.

2 Tsuchiya T, Tabata O, Sakata J and Taga Y. Specimen size effect on tensile strength of surface-micromachined polycrystalline silicon thin films. J. Microelectromech Syst 1998; 7(1): 106-113.

${ }^{3}$ Chasiotis I and Knauss WG, The mechanical strength of polysilicon films: Part 1 . The influence of fabrication governed surface conditions. J Mech Phys Solids 2003; 51(8):1533-1550.

${ }^{4}$ Shikida M, Niimi Y, Hasegawa T, Sugino T, Hamaoka S, Fukuzawa K. Mechanical strengthening of Si cantilever by chemical KOH etching and its surface analysis by TEM and AFM. Microsystem Technologies 2014; 21(3):661-668.

${ }^{5}$ Hajika R, Yoshida S, Makishi W, Kanamori Y, Tanaka S, Esashi M. Mechanical strengthening of silicon torsion bar of MEMS scanning mirror by hydrogen anneal. The 26th International Conference onMicro Electro Mechanical Systems, Taipei, Taiwan, Jan. 20-24 2013, pp.425,428.

${ }^{6}$ Ericson F, Schweitz J-A. Micromechanical fracture strength of silicon. J. Appl. Phys 1990: 68(11):5840-5844.

7 Mitwally M E, Tsuchiya T, Tabata O, Sedky S. Improvement of tensile strength of freestanding single crystal silicon microstructures using localized harsh laser treatment. Japanese Journal of Applied Physics 2014; 53(6S): 06JM03.

8 Miyamoto K, Sugano K, Tsuchiya T and Tabata O. Effect of surface oxide layer on mechanical properties of single crystalline silicon. Mat Res Soc Proc 2008; 1052:53-58.

${ }_{9}^{9}$ Tsuchiya T, Yamaji Y, Sugano K and Tabata O. Tensile and Tensile-Mode Fatigue Testing of Microscale Specimens in Constant Humidity Environment. Exp Mech 2010; 50(4):509-516.

10 Fitch JT, Bjorkman CH and Lucovsky G. Intrinsic stress and stress gradients at the $\mathrm{SiO}_{2} / \mathrm{Si}$ interface in structures prepared by thermal oxidation of $\mathrm{Si}$ and subjected to rapid thermal annealing. J Vac Sci Technol B 1989; 7(2): 775-781.

${ }^{11}$ Uesugi A, Hirai Y, Sugano K, Tsuchiya T and Tabata O. Effect of surface morphology and crystal orientations on tensile fracture property of (110) single crystal silicon. Trans Japan Soc Mech Eng, Pt A 2013; 79 (804):1191-1200.

12 T Yoshioka T, T Ando T, M Shikida M, K Sato K, Tensile testing of $\mathrm{SiO}_{2}$ and $\mathrm{Si}_{3} \mathrm{~N}_{4}$ films carried out on a silicon chip. Sensors \& Actuators: A Physical 2000; 82(1-3):291-296.

${ }^{13}$ Livingston FM, Messoloras S, Newman RC, Pike BC, Stewrt RJ, Brown WP and Wilkes JG. An infrared and neutron scattering analysis of the precipitation of oxygen in dislocation-free silicon. $\mathrm{J}$ Phys C 1984; 17(34):6253-6276.

14 Borghesi A, Pivac B, Sassella A and Stella A. Oxygen precipitation in silicon. J Appl Phys 1995; 177 (9):4169-4244. 


\section{Table and Figure Captions}

Table I Dimensions and nominal Young's moduli of specimens.

Table II Elastic constants of silicon and silicon dioxide used for FEM

Table III Number of nodes and elements of FEM models

Table IV Fracture strains, axial stress in oxide and silicon layer and Weibull parameters. Stress of oxide is at surface and stress of silicon is at the $500 \mathrm{~nm}$ from corner.

Table V Estimated sizes of fracture origin and oxide precipitations.

Figure 1 Single crystal silicon specimen.

Figure 2 Tensile testing system.

Figure 3 Stage displacement-tensile stress plot of a) as-fabricated specimens and b) 200-nm-oxide specimens

Figure 4 Fracture surface of as-fabricated single crystal silicon specimen. Broken line indicate perpendicular cross-section.

Figure 5 Measured nominal fracture strength. Open diamond plots indicate averages.

Figure 6 Fracture surface of single crystal silicon specimen with 50-nm-thick oxide layer. White broken line indicate perpendicular cross-section.

Figure 7 Fracture surface of single crystal silicon specimen with 100-nm-thick oxide layer.

Figure 8 Fracture surface of single crystal silicon specimen with 200-nm-thick oxide layer.

Figure 9 Weibull plots of nominal fracture strength of as-fabricated, oxidized, and oxide-removed single crystal silicon specimen. Lines show fitted Weibull distribution function.

Figure 10 Fracture origin locations. Averages of nominal strength among specimens of each fracture origin are shown.

Figure 11 Fracture surface of oxide-removed single crystal silicon specimen. As-fabricated specimen was oxidized with 200-nm-thick film and oxide was removed.

Figure 12 Simulated axial stress distribution along 45-degree direction from corner applying average tensile stress. Horizontal axis indicates vertical and horizontal distance from corner. 
Table I

\begin{tabular}{lcccc}
\hline & \multicolumn{2}{c}{ Specimen cross section $(\boldsymbol{\mu m})$} & \multicolumn{2}{c}{ Nominal Young's modulus (GPa) } \\
\cline { 2 - 5 } & Width & Thickness & Theoretical & Measured \\
\hline As-deposited & 4 & 5 & 168.9 & 164.7 \\
$\mathbf{5 0} \mathbf{~} \mathbf{m}$ & 4.028 & 5.028 & 164.5 & 168.6 \\
$\mathbf{1 0 0} \mathbf{~ n m}$ & 4.055 & 5.055 & 160.2 & 163.8 \\
$\mathbf{2 0 0} \mathbf{~ n m}$ & 4.11 & 5.11 & 152.3 & 147.5 \\
$\mathbf{2 0 0} \mathbf{~ n m - e t c h e d ~}$ & 3.91 & 4.91 & 168.9 & 174.6 \\
\hline
\end{tabular}

Table II

\begin{tabular}{|c|c|c|c|}
\hline \multirow{3}{*}{ Silicon } & \multirow{3}{*}{ Stiffness matrix } & $c_{11}$ & $165.7 \mathrm{GPa}$ \\
\hline & & $C_{12}$ & $63.9 \mathrm{GPa}$ \\
\hline & & $C_{44}$ & $79.6 \mathrm{GPa}$ \\
\hline \multirow{2}{*}{$\begin{array}{l}\text { Silicon } \\
\text { dioxide }\end{array}$} & \multicolumn{2}{|c|}{ Young's modulus } & 70 GPa \\
\hline & \multicolumn{2}{|c|}{ Poisson's ratio } & 0.17 \\
\hline
\end{tabular}

Table III

\begin{tabular}{ccc}
\hline \multirow{2}{*}{$\begin{array}{c}\text { Oxide } \\
\text { thickness }\end{array}$} & \multicolumn{2}{c}{ FEM model } \\
\cline { 2 - 3 } & Nodes & Elements \\
\hline $\mathbf{5 0} \mathbf{~ n m}$ & 181951 & 20355 \\
$\mathbf{1 0 0} \mathbf{~ n m}$ & 316041 & 35490 \\
$\mathbf{2 0 0} \mathbf{~ n m}$ & 457677 & 51500 \\
\hline
\end{tabular}


Table IV

\begin{tabular}{lccccc}
\hline & \multirow{2}{*}{$\begin{array}{c}\text { Average nominal } \\
\text { fracture strength }\end{array}$} & \multicolumn{2}{c}{ Axial stress (GPa) } & \multicolumn{2}{c}{ Weibull parameters } \\
\cline { 3 - 6 } & $\mathbf{( G P a )}$ & Silicon & $\mathrm{SiO}_{2}$ & Weibull modulus & Scale parameter \\
\hline As-deposited & 4.09 & 4.09 & -- & 5.81 & 4.41 \\
$\mathbf{5 0} \mathbf{~ n m}$ & 3.50 & 3.61 & 1.14 & 5.26 & 3.79 \\
$\mathbf{1 0 0} \mathbf{~ n m}$ & 3.77 & 4.00 & 1.30 & 5.55 & 4.07 \\
$\mathbf{2 0 0} \mathbf{~ n m}$ & 3.27 & 3.69 & 1.16 & 6.42 & 3.50 \\
$\mathbf{2 0 0} \mathbf{~ n m - e t c h e d ~}$ & 2.55 & 2.55 & -- & -- & - \\
\hline
\end{tabular}

Table V

\begin{tabular}{lcc}
\hline $\mathbf{( n m})$ & Fracture origin & Oxide precipitation \\
\hline As-deposited & 19.0 & -- \\
$\mathbf{5 0} \mathbf{~} \mathbf{m}$ & 24.4 & 6.8 \\
$\mathbf{1 0 0} \mathbf{~ n m}$ & 19.8 & 13.7 \\
$\mathbf{2 0 0} \mathbf{~ n m}$ & 23.3 & 27.4 \\
$\mathbf{2 0 0} \mathbf{~ n m}$-etched & 49.0 & -- \\
\hline
\end{tabular}


Fig. 1

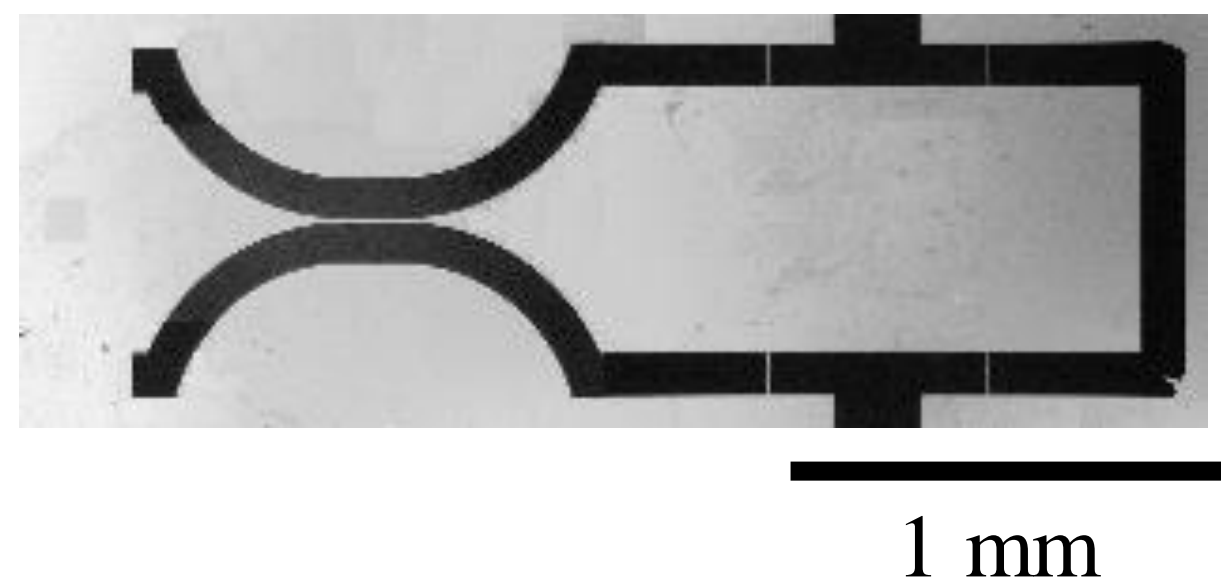


Fig. 2

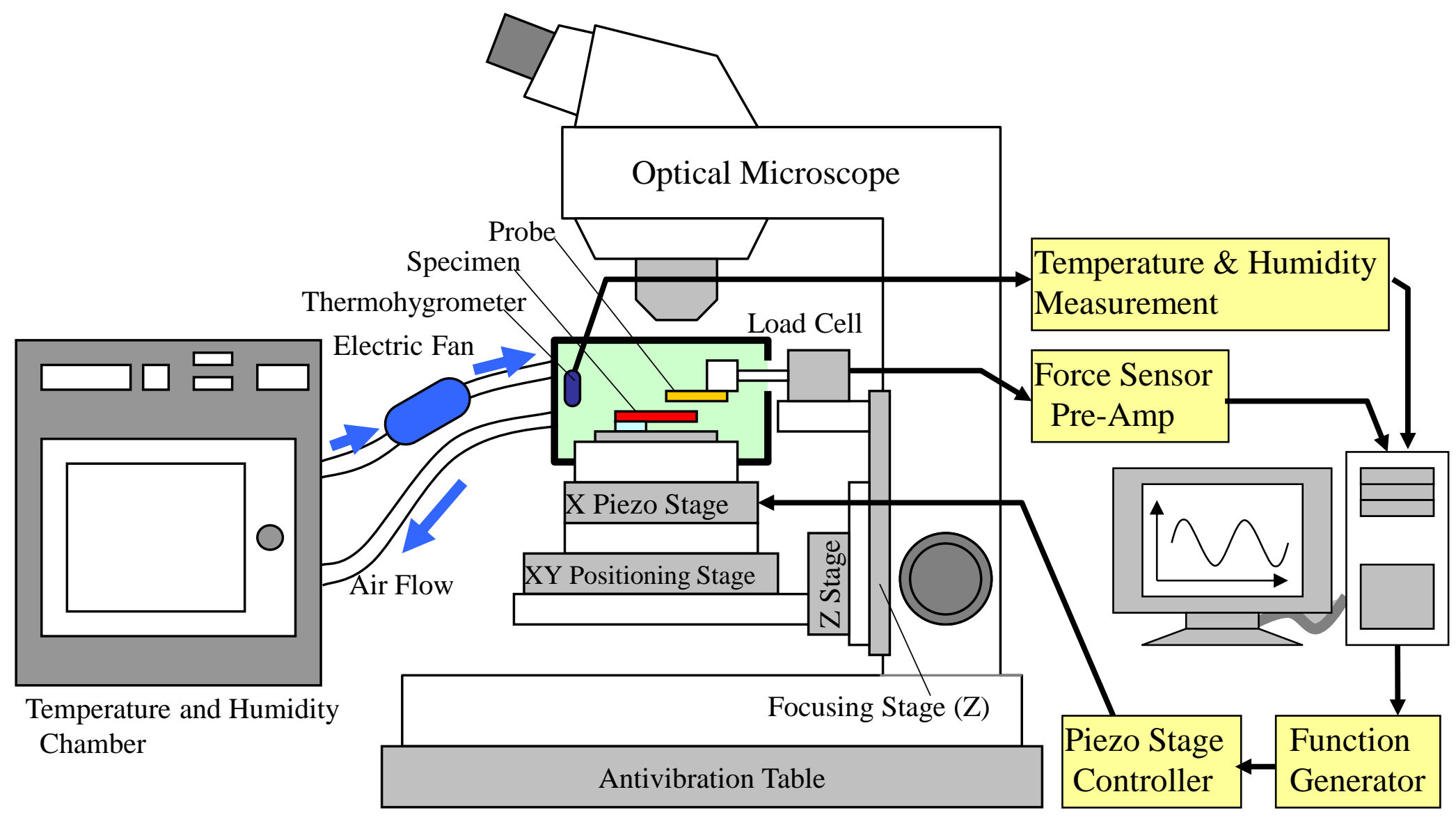


Fig. 3

a)

b)
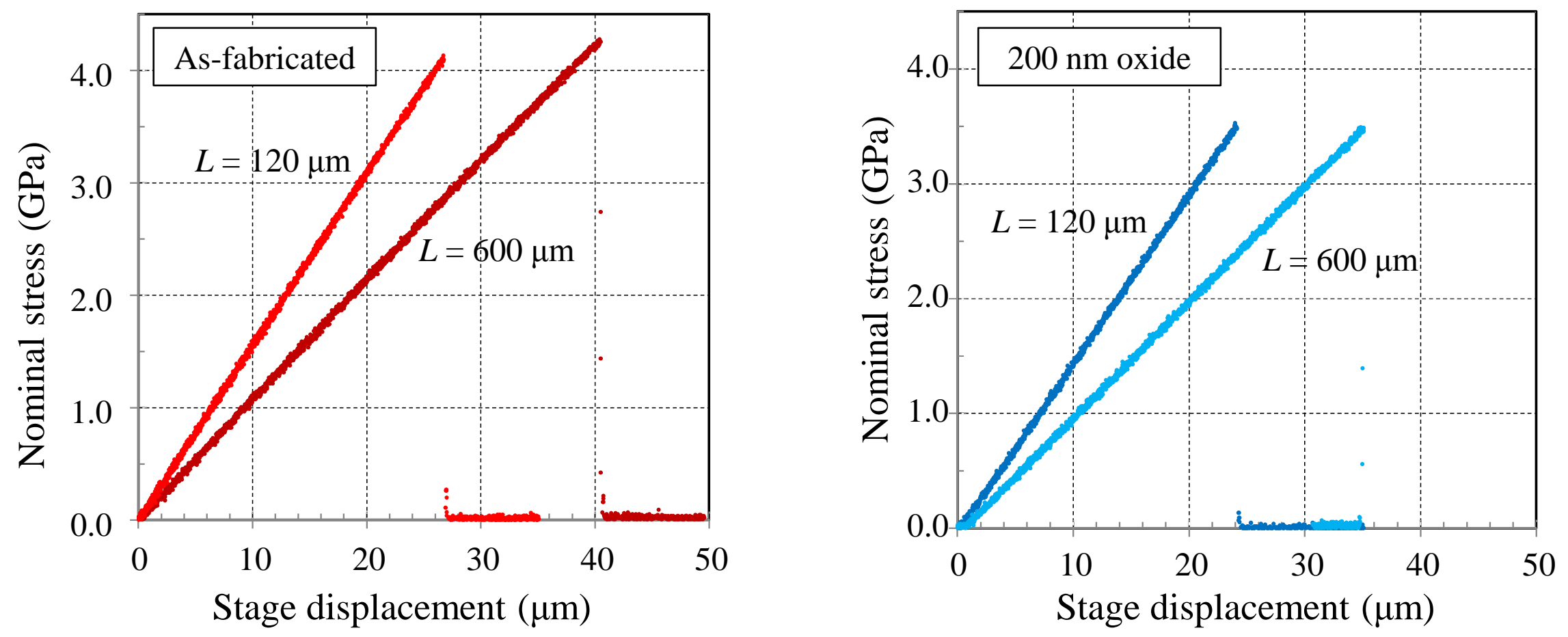
Fig. 4
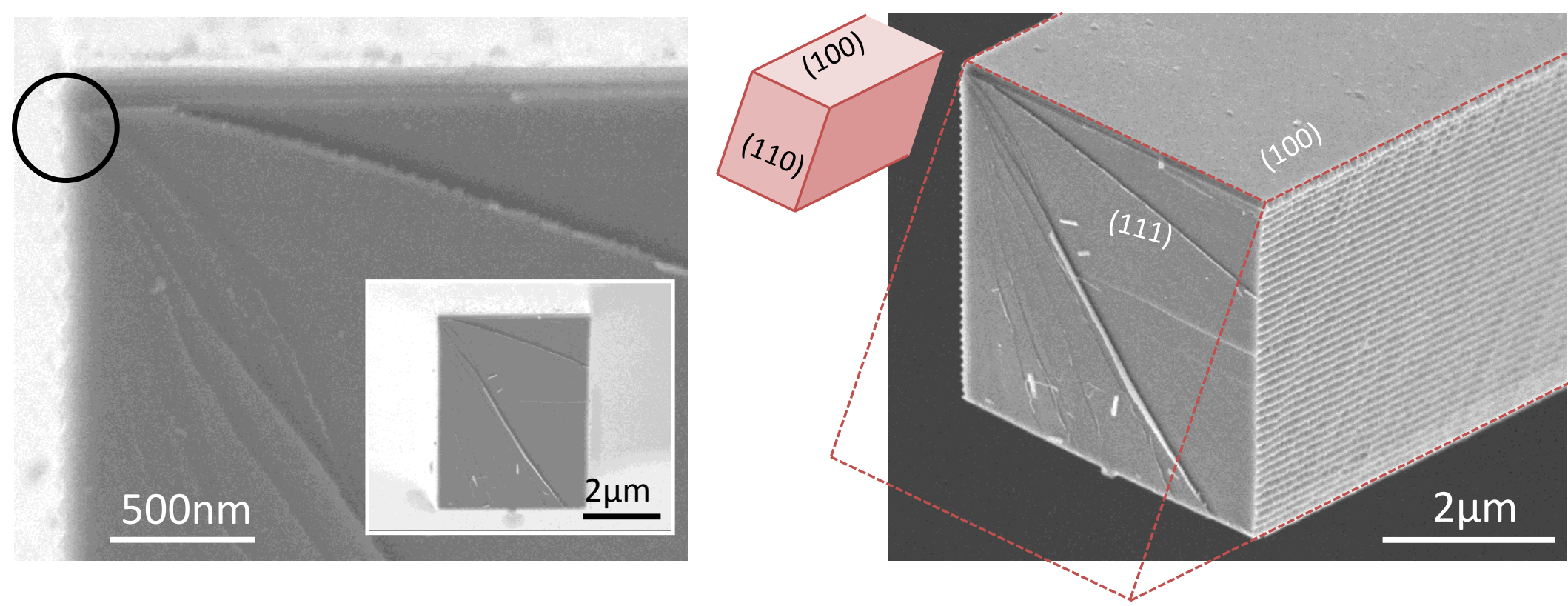
Fig. 5

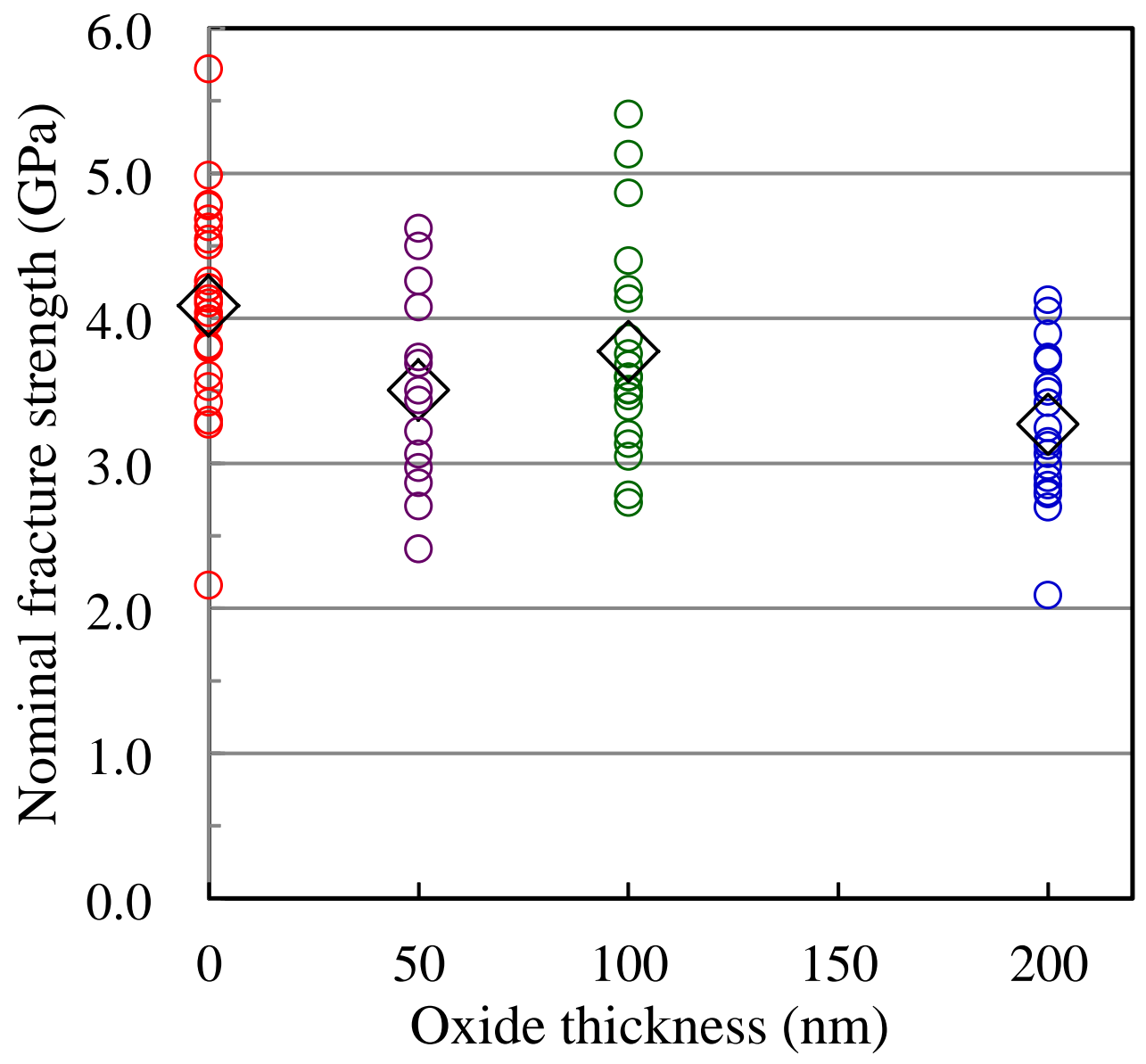


Fig. 6

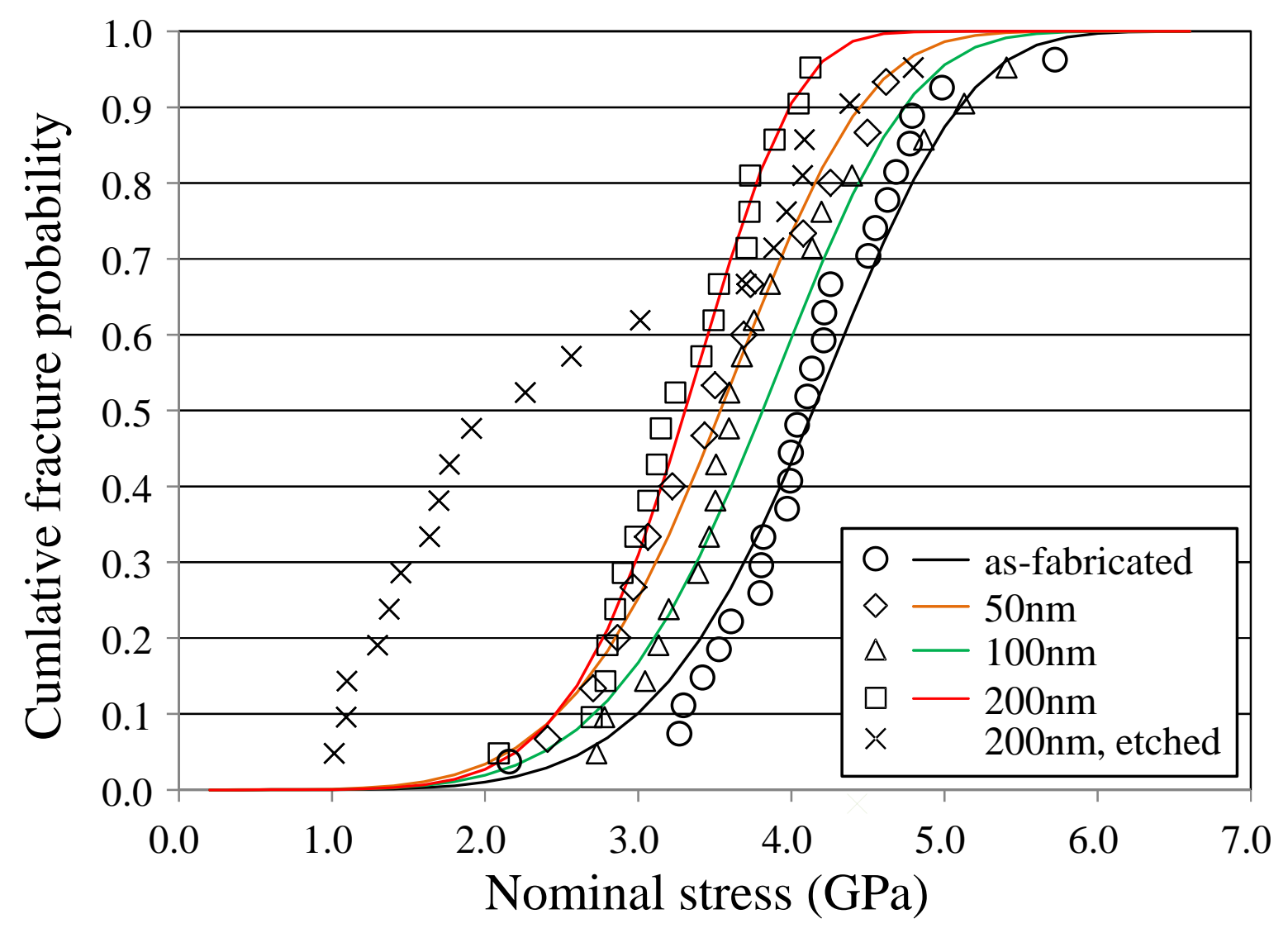


Fig. 7
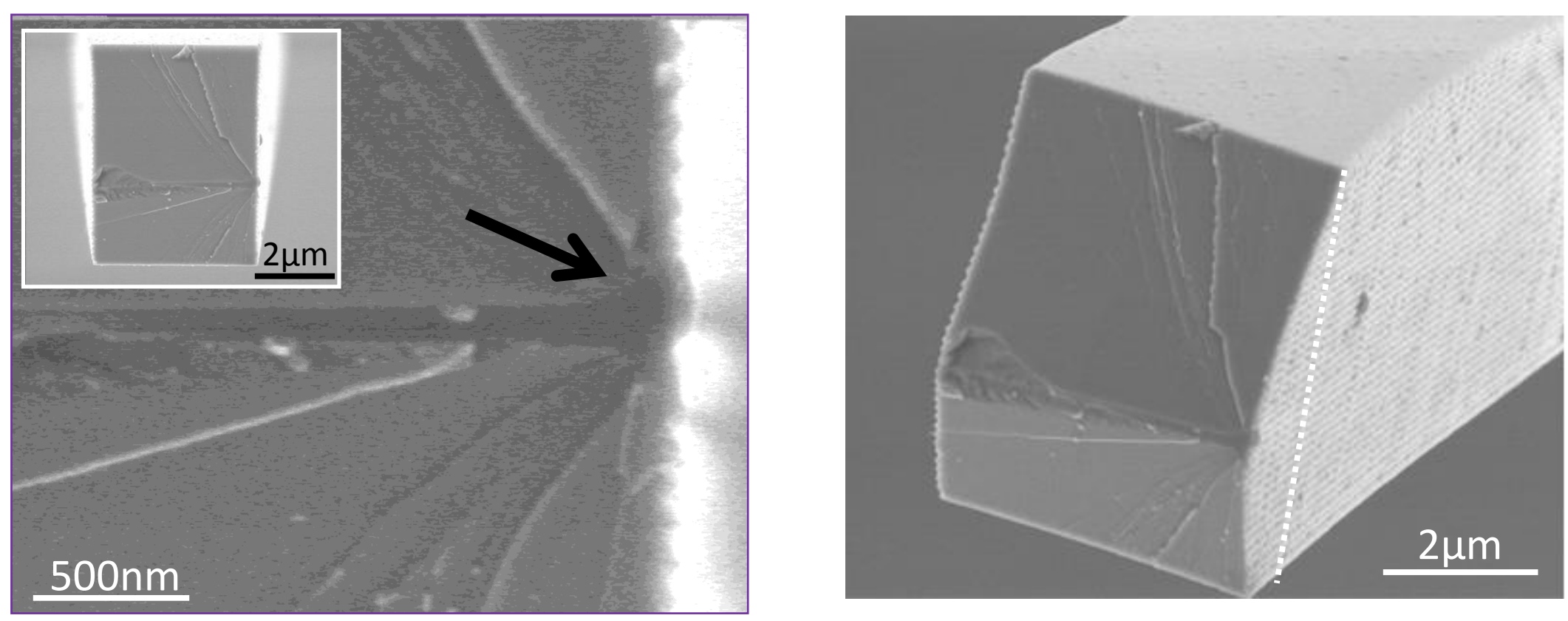
Fig. 8
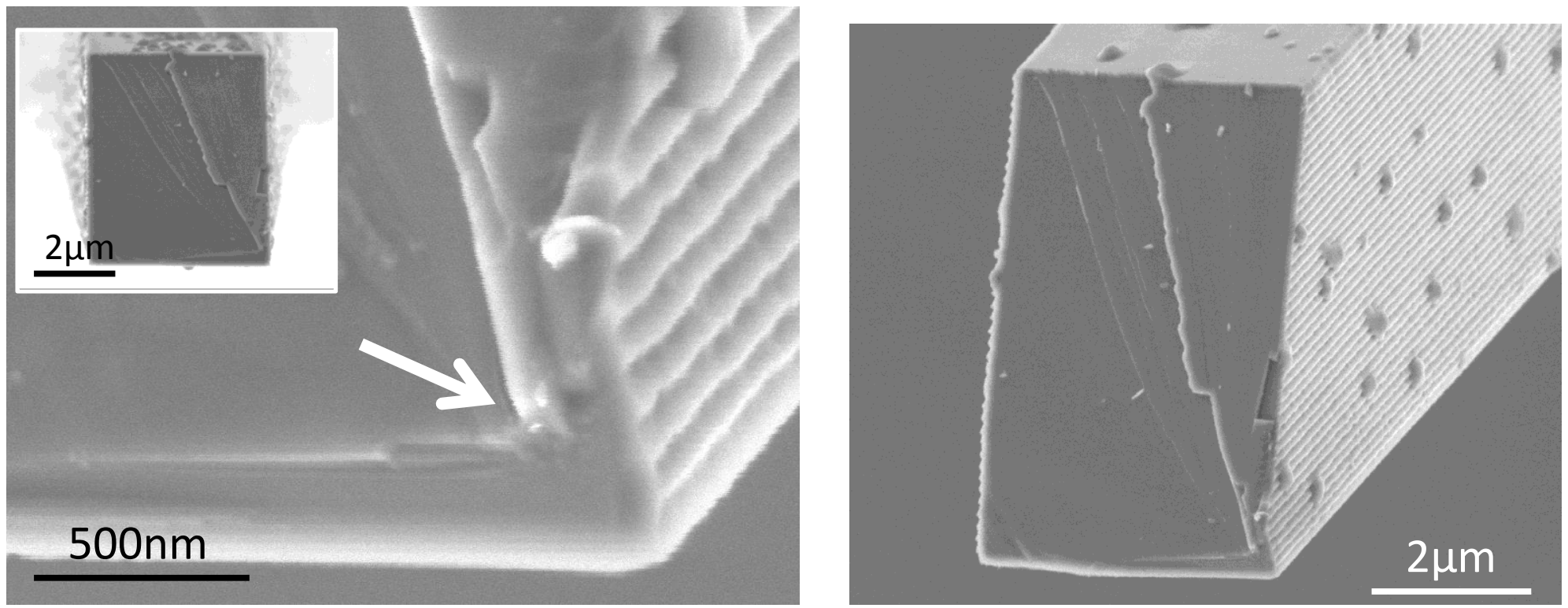
Fig. 9
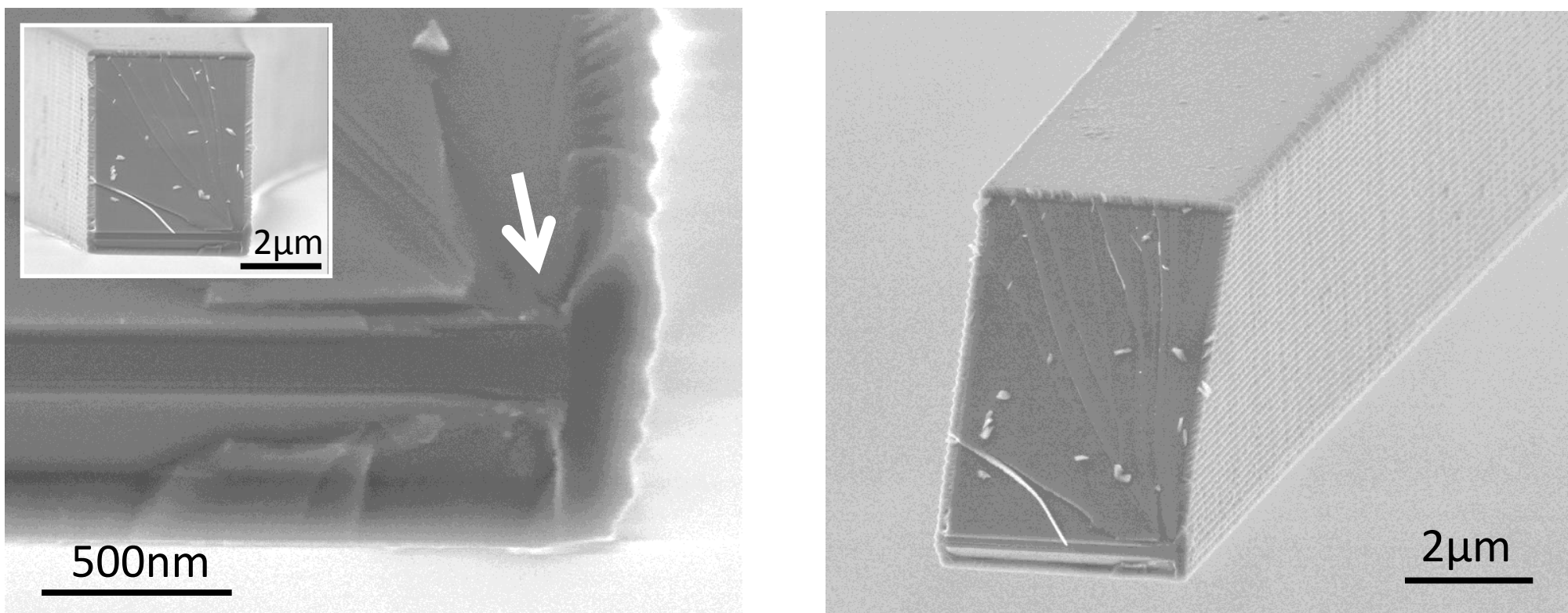
Fig. 10

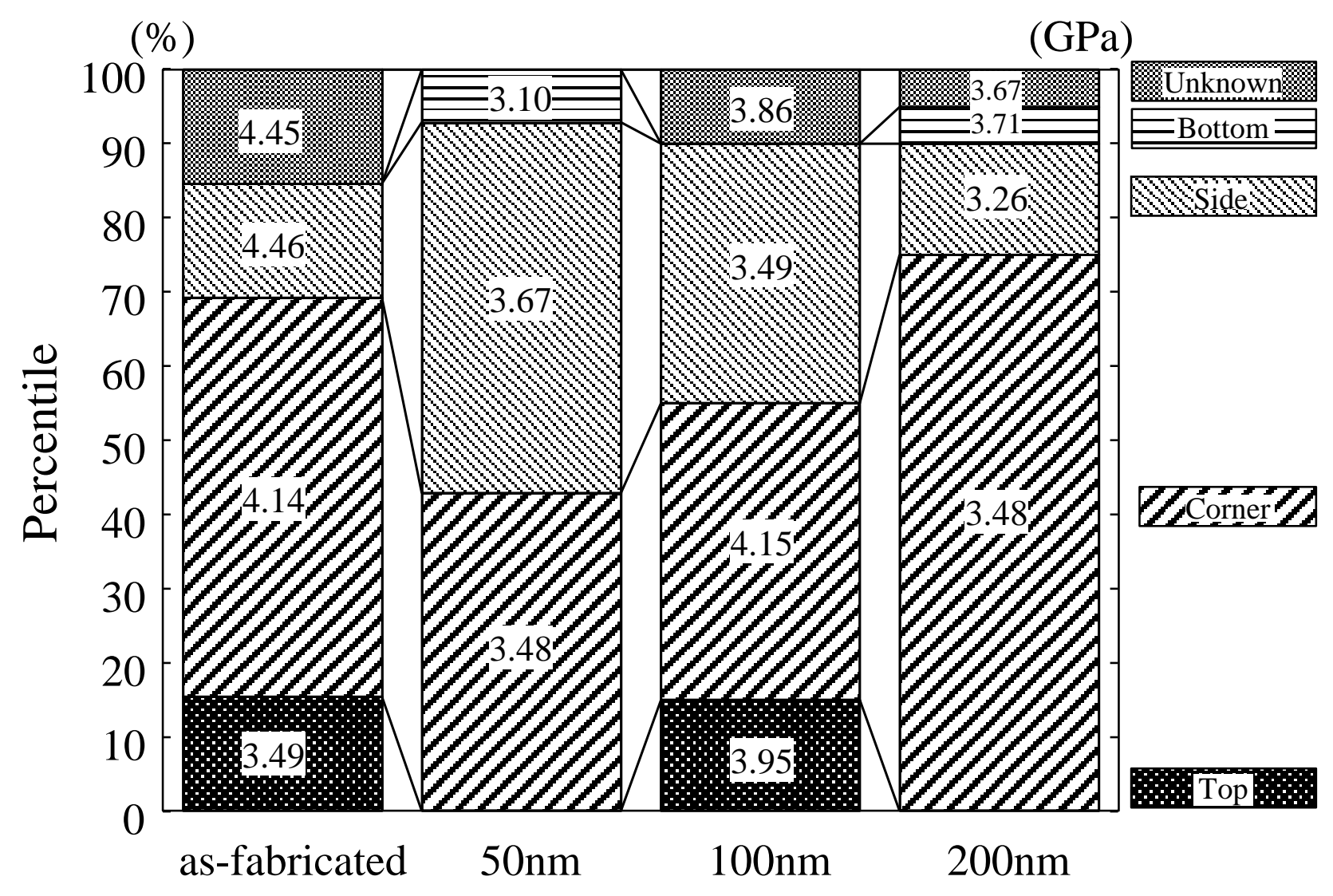




\section{Fig. 11}
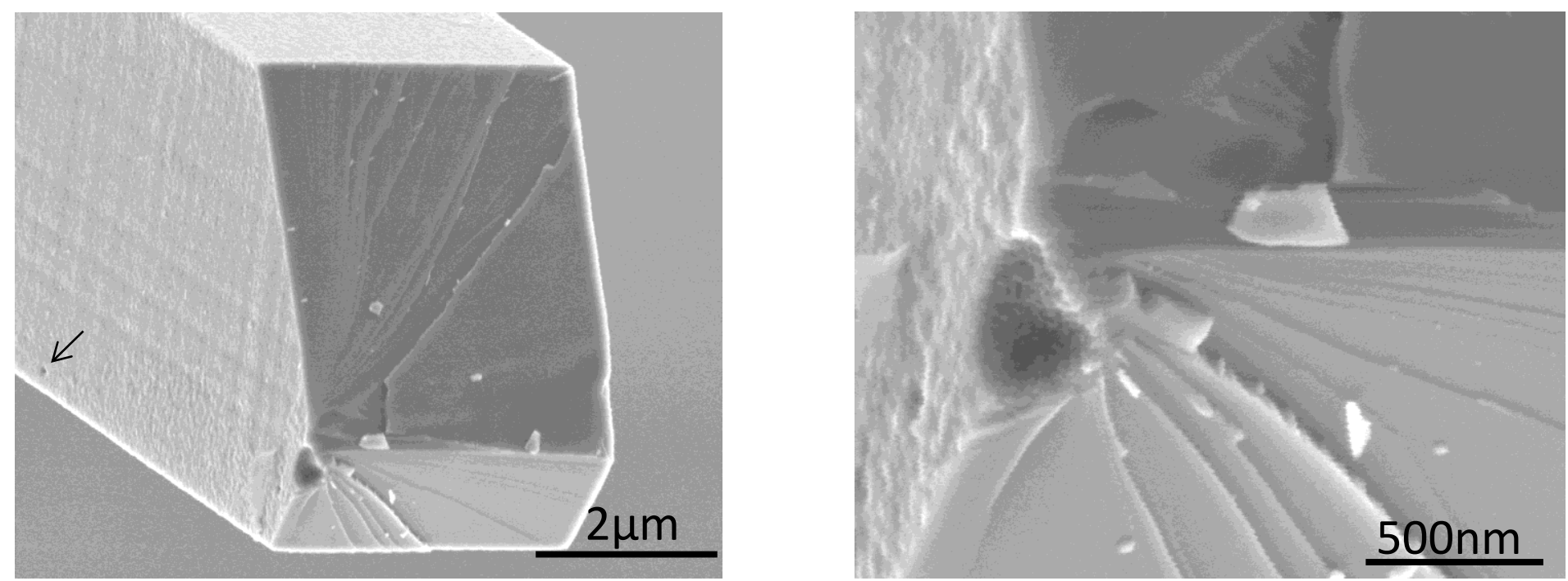
Fig. 12

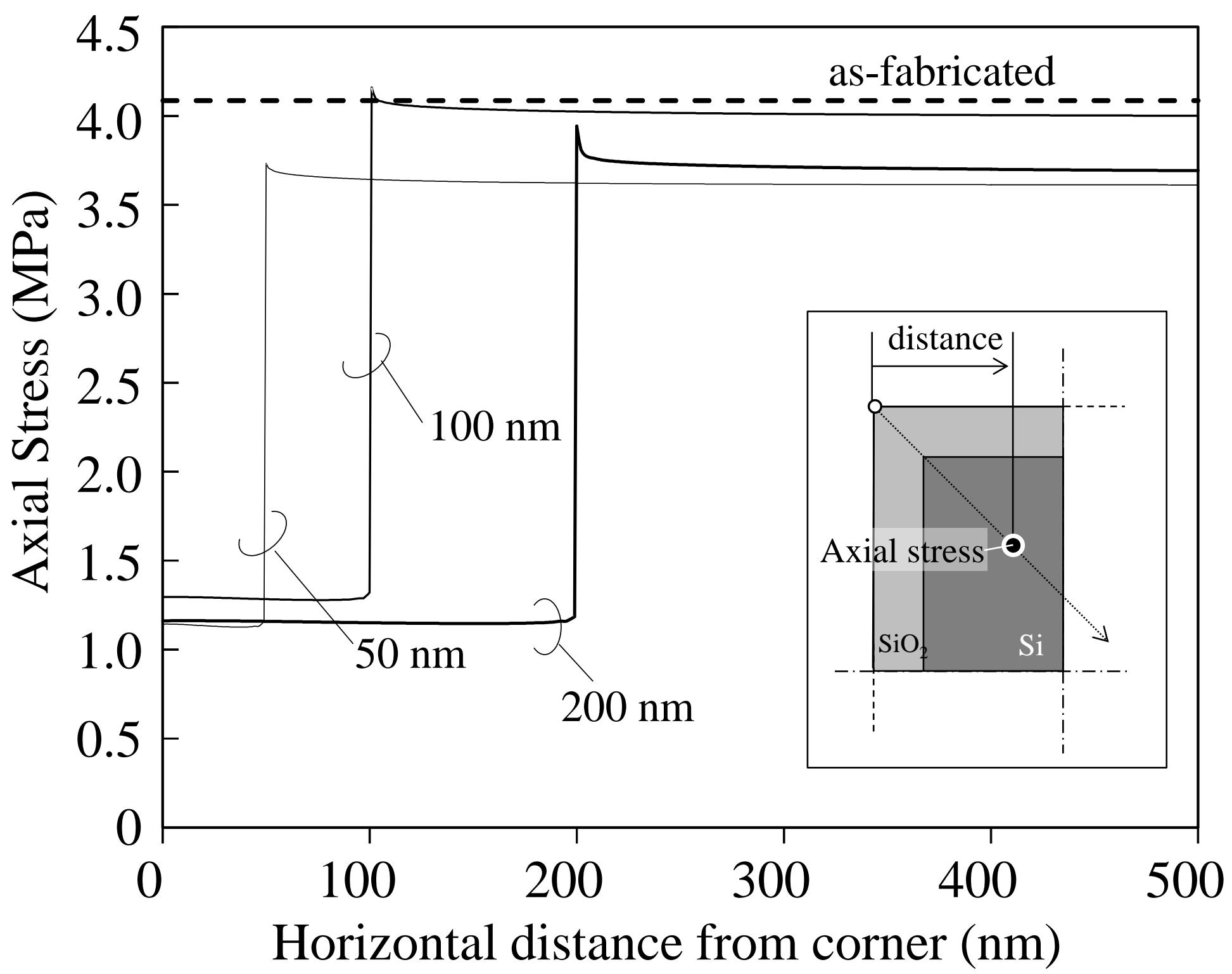

